SANDIA REPORT

SAND99-3117

Unlimited Release

Printed December 1999<smiles>[CH]1[CH]C[CH]1</smiles>

$\because$

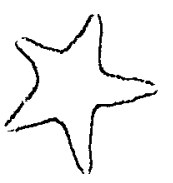

Geom

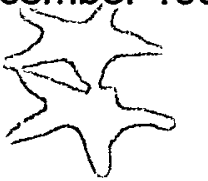

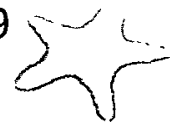<smiles>C=C1C[C]2C=C1C2</smiles>
2

RECFIVED

JAN 102000

OS TI

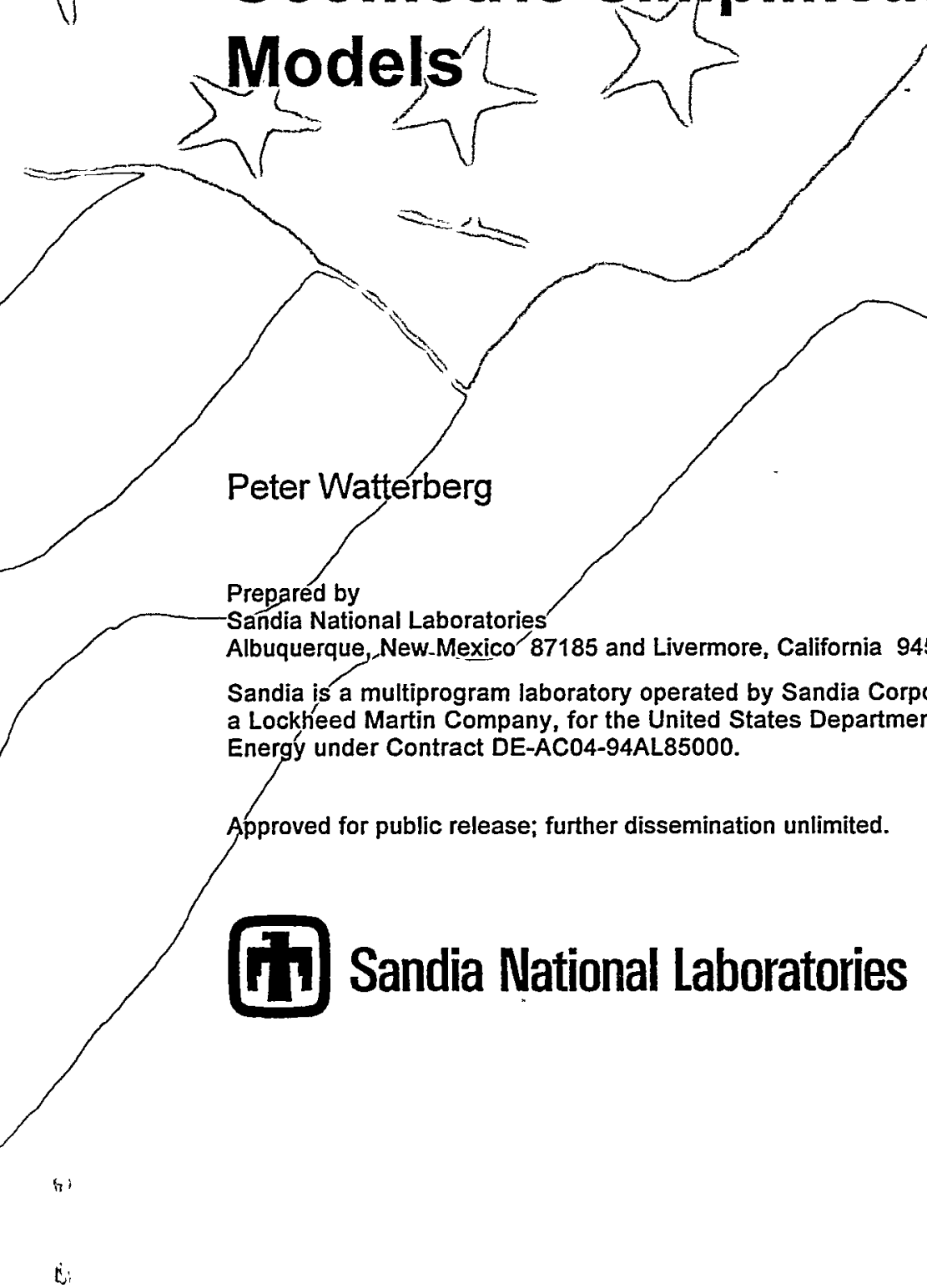


Issued by Sandia National Laboratories, operated for the United States Department of Energy by Sandia Corporation.

NOTICE: This report was prepared as an account of work sponsored by an agency of the United States Government. Neither the United States Government, nor any agency thereof, nor any of their employees, nor any of their contractors, subcontractors, or their employees, make any warranty, express or implied, or assume any legal liability or responsibility for the accuracy, completeness, or usefulness of any information, apparatus, product, or process disclosed, or represent that its use would not infringe privately owned rights. Reference herein to any specific commercial product, process, or service by trade name, trademark, manufacturer, or otherwise, does not necessarily constitute or imply its endorsement, recommendation, or favoring by the United States Government, any agency thereof, or any of their contractors or subcontractors. The views and opinions expressed herein do not necessarily state or reflect those of the United States Government, any agency thereof, or any of their contractors.

Printed in the United States of America. This report has been reproduced directly from the best available copy.

Available to DOE and DOE contractors from

U.S. Department of Energy

Office of Scientific and Technical Information

P.O. Box 62

Oak Ridge, TN 37831

Telephone: (865)576-8401

Facsimile: (865)576-5728

E-Mail: reports@adonis.osti.gov

Online ordering: http://www.doe.gov/bridge

Available to the public from

U.S. Department of Commerce

National Technical Information Service

5285 Port Royal Rd

Springfield, VA 22161

Telephone: (800)553-6847

Facsimile: (703)605-6900

E-Mail: orders@ntis.fedworld.gov

Online order: http://www.ntis.gov/ordering.htm

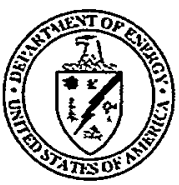




\section{DISCLAIMER}

Portions of this document may be illegible in electronic image products. Images are produced from the best available original document. 
SAND99-3117

Unlimited Release

Printed December 1999

\title{
Geometric Simplification of Analysis Models
}

\author{
Peter Watterberg \\ Intelligent Systems and Robotics Center \\ Sandia National Laboratories \\ P.O. Box 5800 \\ Albuquerque, NM 87185-1004
}

\begin{abstract}
Analysis programs have been having to deal with more and more complex objects as the capability to model fine detail increases. This can make them unacceptably slow. This project attempts to find heuristics for removing features from models in an automatic fashion in order to reduce polygon count. The approach is not one of theoretical completeness but rather one of trying to achieve useful results with scattered practical ideas. By removing a few simple things such as screw holes, slots, chamfers, and fillets, large gains can be realized. Results varied but a reduction in the number of polygons by a factor of 10 is not unusual.
\end{abstract}


This page intentionally left almost blank 


\section{Contents}

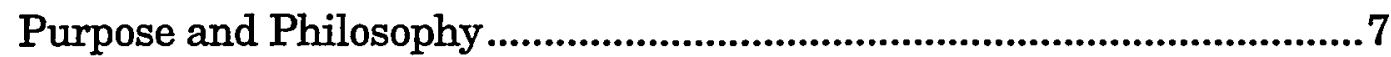

Removing Simple Holes ................................................................11

Removing Arbitrarily Shaped Holes in Planes .......................................15

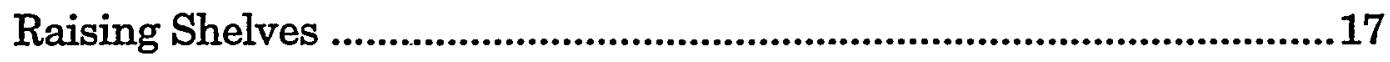

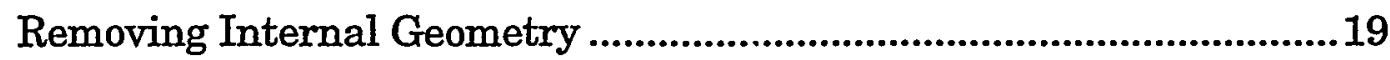

Removing Chamfers and Fillets ......................................................21

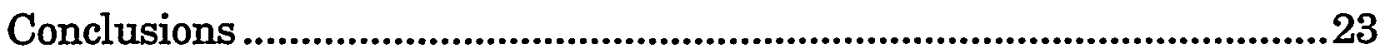

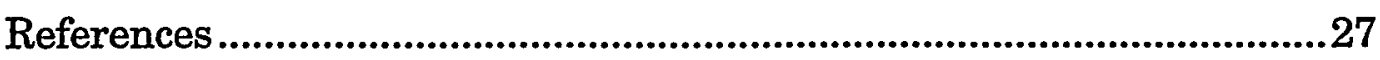

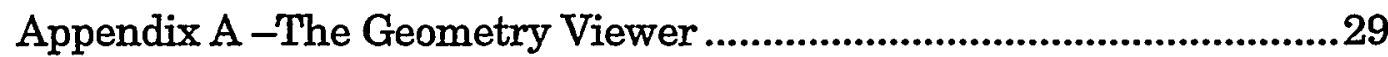


This page intentionally left almost blank 


\section{Purpose and Philosophy}

Most analysis programs, when applied to some object, must deal with that object as a collection of parts. Sometimes the parts are pieces of a higher level geometry such as a torus or spline but more commonly, they are polygons. In either case, the more parts there are, the longer it takes for the analysis to be done. In addition, if the number of parts becomes too large to fit in available memory, the analysis time increases enormously as data is paged back and forth to secondary storage. Of course, there is the possibility that the object description is even too big to fit in secondary, storage.

It is also frequently true that most of the geometry of an object is clearly not pertinent to the analysis. However, very few, if any, analysis programs are equipped to determine which parts of the geometry are pertinent. For example, if the point of the analysis is to avoid hitting the object with a moving robot, then only some simple hull is needed. Or, if the analysis is to determine where to grab the object with either a fixture or a gripper, many things such as screw holes or raised lettering are superfluous. Heat transfer codes probably don't care about the raised lettering either and may not care about chamfers and fillets. And, many codes don't care about internal geometric features.

There has been very little work in the area of removing geometry for purposes of reducing the analysis effort. In the words of Luebke [5], "Very few topologydiscarding schemes have been proposed to date." There has been a number of papers and work in polygon reduction for visual presentation. This work is usually referred to as polygon decimation. The body of work is too large to site here but several example papers are listed in the reference section. This work typically starts with a triangle/polygon mesh or list and seeks to reduce the number of triangles/polygons by replacing several polygons that are nearly coplanar with a single polygon. This work is aimed at reducing the size of data sets of scanned data. None of this work is aimed at actually removing geometric features and changing the topology in order to reduce the complexity and none of it is aimed at CAD models.

Of the work that has been done to remove geometric features for the purpose of simplification, Ames, et al., [1] is the most closely related. They sought to remove features from a Pro/Engineer model to reduce complexity and remove degeneracies. The work had some success but found that working with Pro/Engineer to be too difficult for complete success. Zhuang, et al.[2] extended the concept of alpha-shapes to come up with a hull that mimics the shape of the object better than a convex hull 
but does not preserve any of the actual geometry. This is useful for collision avoidance but not for any analysis that seeks to deal with actual contact or analysis of form.

\section{The Purpose.}

The point of this project is to provide a platform for removing geometry that may be either unnecessary or troublesome. The platform will automatically remove as much geometry as possible (subject to parameters set and heuristics invoked) but will also facilitate manual, interactive removal.

In times past, if an analysis of an object was desired, a model of the object was created (from blueprints or blackboard sketches) just for the analysis at hand. What is more common these days is to receive a request to analyze an object that has been modeled by a separate agency or service. This model will not have been created with the problems of the analysis engine in mind. In fact, since the model was created to satisfy all possible future uses, it is as detailed as the model maker can manage. These models sometimes have the threads of the screw holes modeled and, where the object will have raised lettering such as a part number or manufacturer's symbol, decorative features will also be modeled.

This situation can give rise to polygon counts that are 10 or even 100 times higher than necessary. However, the situation can be much worse than this due to a program's computation complexity. Computational complexity is a rough measure of the running time of a program expressed as a function of the number of inputs. It is usually written $O(f(n))$ where $f$ is the function. It is used to describe how the running time of a program will change as the number of inputs is increased. Thus, if the

program has $O\left(\mathrm{n}^{2}\right)$, then when you double the number of inputs, you will quadruple the running time. If you triple the number of inputs, you will increase the running time by a factor of 9 .

In the case of these analysis programs, we can count the number of polygons or geometric features as the number of inputs. It is not atypical for an analysis program to have $O\left(\mathrm{n}^{2}\right)$. For these programs, if we can reduce the number of polygons it must deal with by a factor of ten, we can decrease running time by a factor of 100 . Potentially, there are enormous gains to be had. Without having done the formal analysis, some programs (Holdfast for one) are thought to be $O\left(\mathrm{n}^{3}\right)$. Early manual attempts to simplify objects led us to hope for a factor of ten on average and we set that as our goal.

\section{Philosophy.}

It is the natural inclination of many, and a matter of history for some, to base this effort to simplify on formalisms or proofs and to choose approaches or 
philosophies that will produce complete solutions. The lack of success using these approaches spurred us to take a more pragmatic tack. We decided to throw a collection of simple, heuristics at individual subproblems and see if we could achieve useful results. This approach also allowed us to decouple all of our ideas. That is, if we had an idea about how to remove a particular kind of feature, an o-ring groove for example, we wouldn't have to spend time worrying about how our idea interacted with other simplification schemes.

Our aim was, then, to create a list of simplifiers which could be sequentially thrown at an object. Hopefully, the list would be somewhat lengthy. Once it was understood what each function did, we could build up groups of functions that would be applied for particular kinds of analyses. For example, heat transfer analysis might be able to safely ignore fillets and chamfers while stress analysis would have to include fillets but perhaps not chamfers. Collision avoidance analysis wouldn't care about fillets, chamfers or internal geometry. As we would identify an analysis type and the kinds of simplifications it needs, we could package them as a group. A user could then request the simplification package to simplify an object for heat transfer analysis or fixture analysis. The simplification package could then be thought of as an automated filter invoked in the progression from design to analysis.

Acknowledging the limits of the spotty approach taken, the simplifier must also enable manual, interactive removal of geometry when automated simplification is inadequate.

\section{Architecture.}

We decided to use the ACIS geometry modeler as the basis for our geometric manipulations. Similar work [1] using Pro/E as the basis had determined that the modeling philosophies and architecture of Pro/E were a great hindrance when attempting simplification work. For example, entire geometric hierarchies may be rooted in a feature that you want to remove. Dealing with these problems was enough of a road block that simplification work never progressed very far. With that experience, ACIS seemed the better choice for this effort.

Furthermore, ACIS seemed to be widely used at Sandia and would thus potentially allow us to have a wide impact. The existence of the Pro/E to ACIS translator would enable us to have some impact on Pro/E models although we would be unable to produce a Pro/E version of the simplified model.

Our scheme for approaching the project was to have a number of independent simplification functions. Each would take as input an ACIS body. Each would then spit out another ACIS body which would have some particular aspect simplified. In this way, we didn't need to have to design an over all architecture for the project. These little simplifiers could be strung together in whatever collection and sequence seemed appropriate for the task at hand. 
This scheme worked for a great deal of the project but it quickly became apparent that there were times when it would be desirable to remove a particular face or feature rather than all of a type of face or feature. To do this, it would be necessary to interactively point it out to our simplifiers. To do this, it was necessary to integrate some functionality with the viewer that was created to help with the visual understanding of what we were doing. In addition, as a convenience, some of the general simplifiers were integrated into the viewer.

It was a given that the only way to achieve any understanding of the composition of ACIS bodies and the effects our algorithms would have on them was to view them graphically. Nothing could have been accomplished if we were relegated to pouring over printed dumps of the ACIS body geometry. On rare occasions, this proved useful but only for painstaking debugging of what was usually a flaw in the ACIS geometry. It was also apparent that the existing functionality for graphically viewing ACIS bodies was far too primitive to be of use. Consequently, we undertook to create a useful viewer. Since we had an extensive background in Motif, OpenGl and graphics, this was not an unreasonable task. The viewer is described in some detail in appendix $\mathrm{A}$. 


\section{Removing Simple Holes}

The simplest feature we could imagine dealing with is a simple hole. By simple hole, we mean a hole who's cross section is a circle or ellipse. So that's what we first attempted. The hole could be a "blind hole", that is a hole that is a dead end, such as a screw hole or it could be a hole that goes all the way through some material, a "through hole", that one might pass a rod or pin through such as the square washer in the figure to the right. This feature was present in a great many examples of geometries of interest.

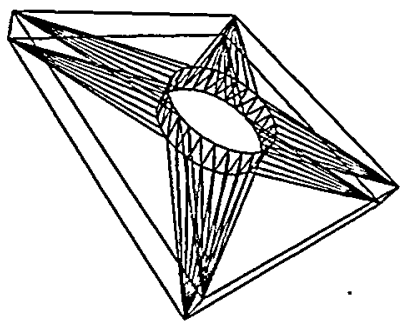
Examining the objects that contained these holes, it was clear that a simple hole in an object generated a large number of polygons.Indeed, punching a hole through an object can generate around 30 polygons in each single polygon the hole pierces and the walls of the hole can be comprised of about 50. These numbers are controllable through parameter settings in the facetter but these numbers are not atypical.

As an example of the difference it can make, notice the result, at right, of removing the hole from the washer. The Archimedes project was asked to do some work with some printed circuit boards and the cage they were placed in. Applying the remove hole simplifier to one of the circuit boards cut the polygon count of the circuit board to one sixtieth, from 24,000 to 400 , without changing the geometry necessary for Archimedes to do its work. The contact analysis portion of the Archimedes run dropped from 4 hours to 6 minutes. Obviously, not all cases will be this suited to hole removal but it was a real case. In another case, applying the hole removal simplifier to a fire set housing, the polygon count dropped $30 \%$.

To find simple holes, we examine all surfaces that comprise a body and pass each through a series of checks. The first check is to see if the underlying geometry is what ACIS calls a cone_type. Not all surfaces of cone_type are simple holes but only this kind of surface can create such a hole. ACIS cones include cylinders of circular or elliptical cross section. If the surface is of cone_type, we must check the sense of the surface to make sure it represents the wall of hole, not the wall of a peg or dowel or some protrusion.

The next test checks to make sure that the surface is a complete cylinder. That 
is, we have to make sure it is not a half of a cylinder split the long way which might represent a groove in a surface, parallel to the surface, rather than a hole through a surface. This represents a problem for the simplifier since a hole can be represented as two half cylinders joined together. In fact, we have seen several examples where the solid modeler, for whatever reason, did just that. The simplifier cannot identify these features as holes. Algorithms for joining the cylinders in these cases have been discussed but not implemented as they were not deemed high enough priority.

The next check insures that the radius of the hole is less than some maximum value. We generally do not want to remove holes of arbitrary size. It is assumed that above a certain size, the hole becomes a significant feature, one that will affect the analysis. The maximum sized hole to remove is specified via a parameter that is settable through the viewer interface.

If the hole passes all these tests, we want to remove it but we must figure out how to treat the surfaces with which the hole intersects. To preserve the validity of the boundary representation of a volume (all of the objects we examine are models of real world objects), we have to close the surface again. If one end of the hole intersects a single surface, it is normally pretty straight forward to remove the hole in the surface that the cylinder makes. In ACIS terms, we simply have to remove the loop that represents the cylinder's intersection. This is still a little tricky since several unwanted things might happen if we just remove the loop.

If the hole in question is a through hole and an end of it is an intersection with a plane, then it is safe to remove the plane's loop and the "hole" in the plane will go away. We can then remove the cylinder. It is possible that the end of the cylinder joins to the end of another cylinder as in two pipes meeting at a joint. If we remove the loop from the other cylinder, it will become infinite in extent. This is a bad thing. In another case, suppose the bottom of a blind hole is slightly spherical in shape. Then removing the loop will create a huge sphere, intersecting with the rest of the body in, probably, disastrous ways.

In general, if the loop of the intersecting surface is the only loop in the surface, then the entire surface is removed along with the cylinder. This is deemed reasonable since the surface is either the bottom of a blind hole or the cylinder is passage into a closed chamber of some sort that will become entirely internal to the object. This might be detrimental to some analysis engines but it would be fairly straight forward to have a flag to indicate whether such a feature should be removed or not.

If the end of the cylinder intersects more than one surface, closing the skin of a boundary representation of a volume can be very tricky. The simplifier makes no attempt at that at this time. Whether the cylinder should be removed and the skin left open depends on what sort of analysis is to be done. If the analysis requires a mesh to be generated, removing the cylinder would not be acceptable. If we are just looking for geometric analysis, such as collisions or contacts or bounding volume 
analysis, then removing the cylinder and leaving the skin open is acceptable.

Once the cylinder is removed (by calling the ACIS routine api_remove_face), the simplifier must begin traversal of all the surfaces in a body from scratch. This is because removal of the cylindrical surface may invalidate some of the pointers in the lists that link all the surfaces.

Specific hole removal. So far the discussion about removing holes relates to removing holes in an automatic manner. It may well be the case that a hole isn't found because it does not satisfy all the conditions necessary (e.g. its radius is too big) and yet it may be desirable to remove it. It may also be the case that the user doesn't want to remove all the holes but a few are causing trouble or obviously hindering the analysis.

In these cases, it would be nice to point to a particular hole and request its removal. The viewer offers just this functionality. This function has been integrated with the viewer since the only practical way to remove a specific hole is to point to it.

When this function is invoked, the viewer asks the user to select a hole by clicking on it. After that, most of the same checks are performed as in the general hole removal (it must be a hollow cylinder, it must be a complete cylinder, ... ). However, the requirement on maximum size is skipped. Also, when removing all the holes automatically in the viewer, a new body is created and put into a new window. When removing a specific hole, the body in which it is selected is modified as it sits in its window. 
This page intentionally left almost blank. 


\section{Removing Arbitrarily Shaped Holes in Planes}

There are many openings, holes, in planes that have the same topology but different geometry as the simple holes described in the preceding chapter. These holes may be slots, o-ring grooves, non-round holes or arbitrarily shaped cutouts. We are looking for cases where this cutout goes through to another plane and there is no complicating geometry along the hole. For example, see the figure 8 cutout at the right. They are harder to find and harder to decide what to do with since they can be composed of many planes and cylinders (and other kinds of surfaces but those holes will be rejected). Consequently, the checks are more complicated.

While not quite as prevalent as simple holes, there are nevertheless numerous examples of this sort of feature. In addition, by noting the number of polygons in the above figure and the number in the simplified picture, the payoff is obvious. The sequence of checks may appear rather complicated as we go through them here. You might feel that innumerable kinds of holes are being rejected for removal. This is true but when a hole passes all the checks listed here,

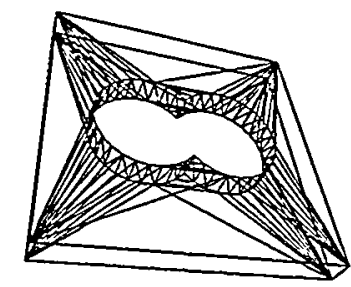
we know what we've got and are confident about removing it.

Remember the philosophy of this project is not to be complete or even very general but to see if we can make a significant difference with some simple, straight forward simplifiers.

To simplify an object in this way, we step through all the surfaces of the object looking for planes in which we can patch holes. In looking for simple holes, we looked for cylinders that might be holes. Here, we are looking for planes that might have holes in them. The same sorts of checks used here might work for other kinds of surfaces. We didn't feel, however, that we could confidently understand all the ramifications and possibilities for other types of surfaces. Consequently, the approach is to traverse the faces in the ACIS body looking for planes. If we find one, we examine every loop in it. If it is an (the) external loop, it is passed over.

Next, we compute the length of the loop, or the length of the edge of the hole, if you will. We will use this analogously to the radius of the simple hole to determine if this feature is small enough to be removed. Another option might be to determine 
the area of the hole and use that for screening but the edge length is directly related, easy to come by and seemed useful enough for now.

Next, all the faces that comprise what might be the wall of the hole are found by looking at the faces that share the edges of the loop (in ACIS terms, we get the faces that contain the coedge partners). Each of these faces is checked to make sure there are no other features on the face. We do this in ACIS by making sure the face has only one loop. This assures that there is no interconnecting geometry.

Next, we find the surface at the other end of the hole. We make sure it is a plane. Further analysis might allow the inclusion of some other surfaces here but, while conservative, allowing only planes still includes a large fraction of the kind of feature we are trying to remove. We also have to make sure that all the faces that comprise the hole are adjacent only to each other and the planes at the ends of the hole.

Finally, we have to make sure that all of the surfaces of the hole constitute a structure that penetrates into the body rather than rising above it. If this is the case, we have found a structure to remove. We remove all the surfaces of the hole and the the outline of the hole in the first plane (the ACIS loop). If the second plane faces the same way as the first plane, then it must be the bottom of a blind hole, perhaps the bottom of some recessed indent. If so, we must remove the face, otherwise, we must remove the loop in the second face that is the outline of the hole.

Removing this hole means that ACIS will create fewer polygons when faceting the object. Most analysis programs deal with objects as a collection of polygons and thus will have fewer things to deal with.

In addition to the automated removal of planar cutouts, it can be useful to select specific cutouts for removal. This works just like the specific hole removal. The user selects a cutout by clicking on the wall of the cutout. The size restriction used to filter automated plane patching is disregarded when doing interactive plane patching since it is assumed that the user is directly in control and is aware of the size of the cutout. 


\section{Raising Shelves}

There are many occasions when a relatively large flat surface is lowered in one section to accommodate something such as an o-ring groove or an indent for a bolt head. If this happens in the interior of the flat surface, it .. . will probably be found if the plane patcher is invoked. However, many such occurrences are on the edge of the plane such as in the figure to the right. These cannot be found as a hole in a plane but they are common enough and expensive enough that we would like to remove them. If the

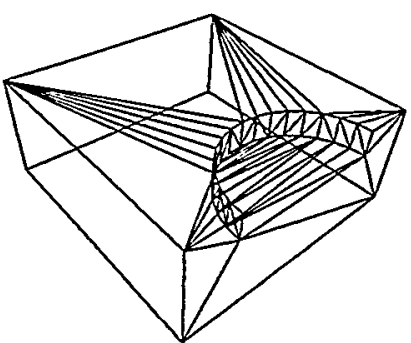
shelf is only a little lower than the main plane and the geometry between the two is not too complicated, we would like to raise the shelf, patch it together with the plane and remove all the geometry between the two.

The two features of interest in this case are related by proximity (the distance between the two planes must be less than a user specified parameter) and orientation (we require that the face the same way, i.e. the dot product of the normals of the two planes be 1.). Consequently, we can't look for a single feature with some characteristics.

The search for shelves begins by finding every plane

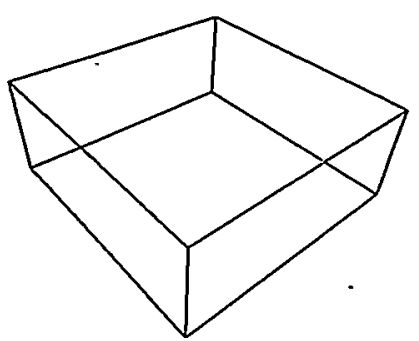
surface in the body and creating lists of planes that face the same direction. As indicated above, this is a simple dot product check of the plane normals. For each list of planes that face in the same direction, the planes are sorted front to back relative to the direction they are pointing.

The next step is to begin at the farthest plane (if you picture stacking the planes vertically with them all facing up, this would be the highest plane) and see how far it is to the next lower plane. If the distance between the two is greater than the maximum allowable distance (a settable parameter), the lower plane is not a candidate for raising.

Since we are going to raise the lower plane and we don't want to change the topology of the object, we need to make sure that all the faces bordering the lower plane are perpendicular to it. That way, any face that is between the upper and lower planes will be removed and any other face will simply be extended. It is conceivable that the lower plane could be successfully raised even if the faces between the two 
were not perpendicular but such analysis looks to be more difficult than is warranted by the potential payoff. Even if all the faces are perpendicular, we must also make sure that there is no geometry between the plane we are raising and the level to which we want to raise it. If there is, we could create pathological or non-sensical geometry.

A final condition that must be met is that there exists a face that is adjacent to both the upper face and the lower face. This is necessary so that the two faces can be stitched together after the raising. If they are not contiguous after the raising we haven't reduced the geometric complexity.

Once these conditions are met, the shelf is raised and merged with the upper plane. This is done is ACIS by making a new body and uniting it with the old body. The new body starts as a copy of the face that is the shelf. It is then swept through a distance equal to the difference between the two planes. This body then represents exactly the volume that we want to fill. This is then united with the original body, relying on ACIS to do the boolean correctly. 


\section{Removing Internal Geometry}

By internal geometry, we mean geometry that is not accessible directly from the outside. This may be features that are truly, topologically internal such as a hole in the middle of a block as in the figure to the right. In addition, this includes geometry that may topologically share the same surface as the outer surface but is only connected in some serpentine manner. For example, every wall in a maze is part of the same surface as the outside of the maze but

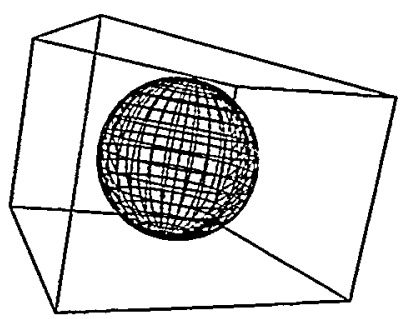
almost all the geometry is internal. There are many applications that are not at all interested in internal geometry. Any sort of collision or contact analysis is much quicker if the internal geometry is removed.

This is the one simplification method that requires the use of a graphics system. Since the viewer was already in place, it was fairly straightforward to use the graphics environment that was already set up. The algorithm is almost identically analogous to the following: Hold the object in front of you and color everything you can see blue. Turn the object a little bit and repeat. Keep doing this until everything you can see is blue. Then, as if from a 4th dimension, look inside and remove everything that wasn't painted blue. Using this method, all the surfaces that may be encountered by anything else that is going to approach this object are still there. Thus any analysis that only cares about this sort of interaction will still work.

To implement this technique in the computer, we take advantage of the graphics engine. It can render a view of the object in fractions of a second. In each view, it has to determine exactly what is visible from the given viewing orientation. This is usually done with a Z-buffer. Normally, after figuring what is visible at a pixel, it figures out what color should be displayed by combining the object's color with the current light source colors and ambient light. For purposes of determining what is visible and retrieving that information, we can assign a unique "color" to each face (an integer between 0 and the number of faces) and turn off all lighting effects.

We can then render the picture into a non-viewable buffer. Then we read back all the pixels and look at the color value. The color value is used as an index into an 
array representing all the faces of the object. We initially mark each face as invisible. When we find one in the image, it is marked as visible. In this way, we find all the faces that are visible from a given orientation. If we repeat this for a number of "good" orientations, we'll build a list of all the faces that are visible from somewhere. It's a simple matter after that to remove all the faces that weren't visible.

What's left is to select the viewing orientations. We start by collecting all the normals of all the planes. A view is drawn for each unique normal. A view is also drawn along the axis of every cylinder. The drawings are done with an orthographic projection rather then the perspective projection that is normally applied for viewing the object. The distortions of a perspective projection can hide surfaces.

After each view is drawn, all the pixels in the rendering buffer are read back. Each pixel is examined to see what "color" is there. If it is not the background color, it is the color of a particular face. The face index is reconstructed from the red and green components of the color. The index is used to mark the face as visible. If one could be sure that there were no more than 255 faces, the rendering could be done in a 8 bit buffer (a very common size) and the index would not need to be reconstructed from two color components. This would speed the process up a bit.

After all the views are rendered, the list of faces is examined and every face that is not visible is removed from the body. The removal is done directly on the body as it sits rather than making a copy of the body. 


\section{Removing Chamfers and Fillets}

Whenever two planes come together on a real world object, they almost always have some chamfer or fillet . blending the two surfaces. If they meet in a convex, outside corner they will have a chamfer as shown here. If it is a concave, inside corner, it will be a fillet. Frequently, the blend will be large enough to be worth noting in the design. Often the blend is a natural result of the manufacturing process but sometimes it is explicitly designed in. These blends are always curved surfaces and it is curves that cause the polygon count to go through the roof. In the example here, the polygons increased by a factor of 10 when adding this chamfer.

Finding chamfers begins by finding cylinders. We look for . cylinders that have circular arc cross sections. The cylinder must then have only one loop. This ensures that the cylinder isn't a hole or rod. Then we check to see if it joins two planes along its straight edges. If a cylinder represents a simple chamfer, it will have four edges, two straight edges along the two planes it joins and two other edges. The two other edges may connect with surfaces that are planes, spheres, tori, cylinders or splines. We can do something reasonable with anything but splines. At this point we require that each of the planes have only straight edges preceding and following the edge that runs along the chamfer. With one exception, any other kind of edge is too difficult to deal with at this point.

If the chamfer candidate gets this far, we compute the new geometry needed and attempt to modify the ACIS model. We can compute the line segment and vertices in which the two planes will meet. We can then modify the appropriate pointers in the ACIS model. The difficulty is in accounting for all the possible conditions at the end of the cylinder. This involves special casing the different possibilities.

In the simplest case as shown in the above figures, the surfaces at the end of the cylinder are planes. To deal with this, we throw away the circular arc and adjust the two edges to end at the new vertex that has been computed. If the end of the chamfer abuts another chamfer, then the one plane will have to have a circular arc 
added to its corner and the abutting chamfer will have to be modified. When this is done we will get back to the simple case explored first. It can then be handled that way when the second chamfer is up for consideration.

If the end of the chamfer abuts two other chamfers, the three can come together in a point or they can be blended together with a sphere or a torus. The torus is used when one of the chamfers has a different radius than the other two. We do not handle the case where they come to a point. If they are blended by a torus or a sphere, the torus or sphere is removed and the geometry is calculated to result in the second case talked about above.

The work for fillets should proceed along very similar lines. However the investigator that did this work left the company before doing that work or writing a report on chamfers. Hence, this report less detailed than it would otherwise have been. 


\section{Conclusions}

The result of throwing this hodge podge collection of tricks at an object has been fairly satisfactory. We had set as a goal a 10 to 1 reduction in the number of polygons. For the most part, we achieved that goal. The factors ranged from 60 to 2 for various of the samples we tried it on. As was mentioned in the section of removing simple holes, the factor of 60 was achieved on a circuit board that had been drilled with many little holes for the wires and ICs.

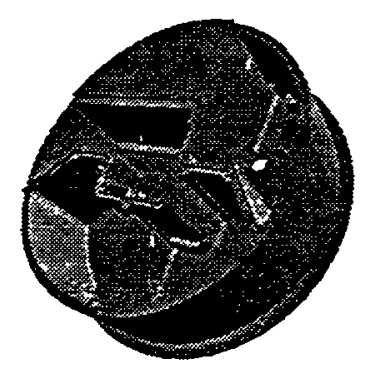

The object that showed the least improvement is shown above. The reason it only reduced its polygon count by a factor of two is explained in that it is inherently a round object. It is precisely round or curved surfaces that require many polygons to approximate their shape. Objects that responded the best had little or no rounded basic structure but did have many rounded details such as holes and chamfers. A more typical object is shown below. The side by side views show that although the polygon count was reduced by a factor of about 9 , the essential shape appears the same.
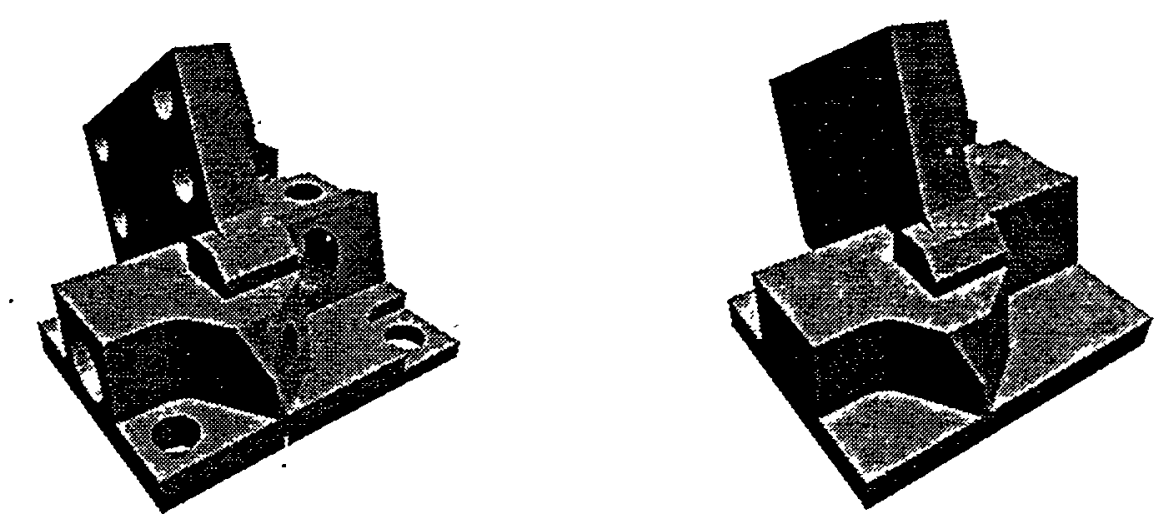

At the top of the next page the same two views of the object are rendered in 
wire frame. This gives a feel for the reduction in the number of polygons.
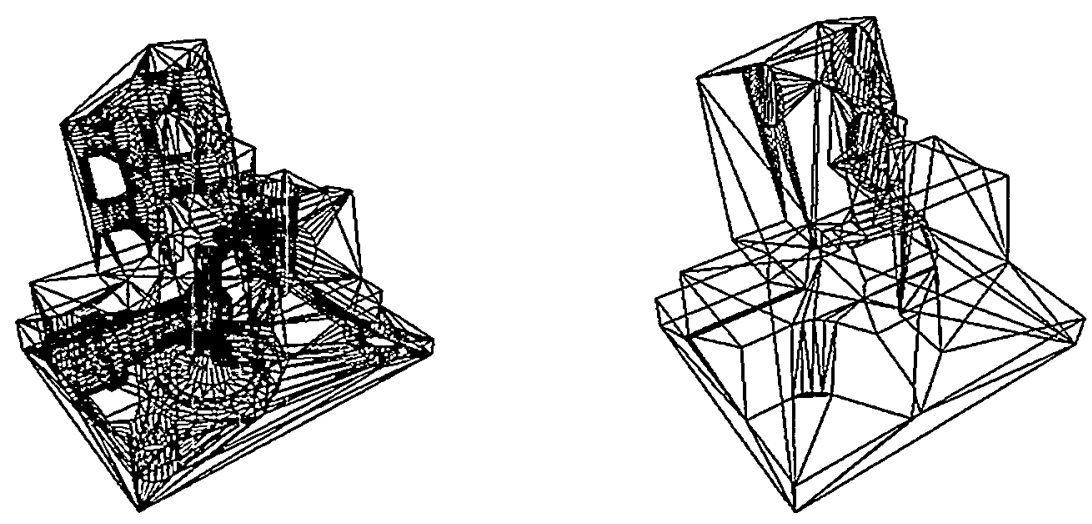

One important thing to note about throwing this bag of tricks at an object is that the final result can be highly dependent on the order in which things are applied. The hole remover may not find a hole until the chamfer remover has straightened up the edges. The internal geometry remover may have very different results depending on whether a hole that looks in on it has been closed or not. Also, a hole in a plane could be found and patched by either the hole remover or the patch plane simplifier. These two different simplifiers may do something different with the result.

Another question one has to ask when using these techniques is what kind of object is produced, that is, what is the topology of the resulting object and what is its volumetric relationship to the original. It is possible when selectively removing holes or patching planes to cause the simplifer to break the boundary representation of a solid. At the moment, the simplifier assumes the user understands the implications of each request made and removes the feature even if it can't maintain the boundary representation. This means that the object no longer represents a potentially realizable solid object. It may have more in common with a Klein bottle where inside and outside are not distinguishable. This defect is not a problem for geometric analyses such as collision avoidance or grasping or dynamic interactions. It will be a problem for analyses that require a sane, solid body such as thermal or stress analysis or anything that is to be analyzed using something like finite elements.

A nice addition to the simplifier would be a flag that the user could set that would require the simplifier to maintain the boundary rep and only make simplifications that would satisfy this condition. This will have to wait for the next version.

Another characteristic which should be factored into the simplification is the simplified object's volumetric relationship to the original object. For example, do we require the simplified object to be a superset of the original? That is, does it need to be some sort of enclosing hull? In fact, one way to do a simplification is to create the convex hull of the object. This would be straightforward with available routines but wasn't thought to be very useful for most analysis. However, for an analysis such as 
collision avoidance, we want to be sure that the resulting object is an enclosing hull of the original. Analyses such as thermal or stress probably don't care much about this property.

In general, we were successful in achieving the kinds of polygon count reductions we were aiming for. We regard this as a proof of concept and there is a great deal to be done to make this useful as a general tool. There are many more heuristics that could be developed. A lot of energy could be devoted to relaxing the fairly tight restrictions on the heuristics that we do have. We had to make them very restrictive so that we could get somewhere. By relaxing them, one could encompass more kinds of geometry.

It would also be nice to group heuristics and parameters into sets that were appropriate for particular applications. For example, a stress analysis application does not want fillets removed but probably doesn't care about chamfers. It also wants the body to remain solid. If these application packages were well honed, it would be possible to simply ask the body to be simplified for stress analysis, or collision avoidance problems or whatever. 
This page intentionally left almost blank. 


\section{References}

[1] A. L. Ames, J. J. Rivera, A. J. Webb, D. M. Hensinger, "Solid Model Design Simplification”, Sandia Report SAND97-3141, 1997.

[2] Y. Zhuang, K. Goldberg, M. Pickett, "Simplifying Complex CAD Geometry with Conservative Bounding Contours", Proceedings of the 1997 IEEE International Conference on Robotics and Automation, April 1997.

[3] W. J. Schroeder, J. A. Zarge, W. E. Lorensen, "Decimation of Triangle Meshes", Computer Graphics, Vol. 26, No. 2, July 1992, pp. 65-70.

[4] T. Lu, J. I. Khan, D. Y. Y. Yun, "An Optimizing Algorithm for 3D Object Surface Triangulation", Proceedings of the IASTED International Conference, August 19-21, 1996.

[5] D. Leubke, "Hierarchical Structures For Dynamic Polygonal SImplification", Technical Report 96-006, University of North Carolina at Chapel Hill, 1996.

[6] T. He, L. Hong, A. Kaufman, A. Varshney, S. Wang, "Voxel-Based Object Simplification", Proceedings Visualization 95, IEEE Computer Society Press, 1995, pp. 296-303. 
This page intentionally left almost blank. 
Appendix A

The ACIS Geometry Viewer 
This page intentionally left almost blank. 
The only reasonable way to understand the composition of ACIS bodies and the effects of our algorithms on them was to view them graphically. Attempts to analyze printed dumps of body geometry were immediately rejected as being totally incapable of delivering insight. This method of analysis was only useful in painstaking debugging efforts. The existing functionality for viewing ACIS bodies graphically was far too primitive to be of use. So, we undertook to create a useful viewer. Since one of us had an extensive background in Motif, OpenGl and graphics, it was a reasonable task to undertake and the results are satisfactory.

\section{Architecture.}

The viewer is based on Motif and OpenGl. ACIS bodies are generally composed of analytical surfaces. OpenGl is only able to display polygons. Consequently, when an ACIS body is loaded for viewing, the ACIS facetter is invoked to decompose the body into polygons. A custom mesh manager was written for ACIS which receives the polygons as the ACIS facetter creates them. A separate display list is created for each ACIS face. This allows displaying different faces with different visual attributes. This is important when trying to determine how the different faces fit together to form the ACIS body.

The interaction and presentation of the viewer is somewhat minimalist in philosophy. The initial interface (Figure A-1) consists of a single menu bar with five

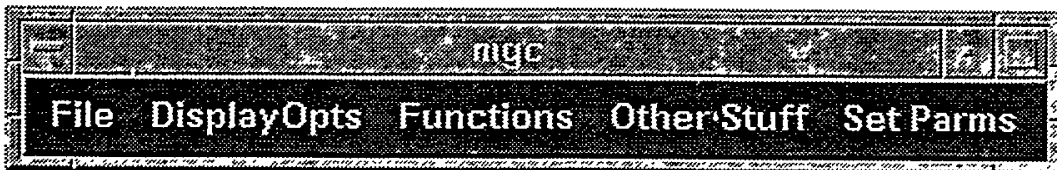

Figure A-1

buttons representing different groupings of actions. When a body is brought into the viewer, a window is created in which to view it. Any number of bodies can be brought into the viewer and each is given a separate window. Viewing interaction is primarily mouse driven. Pan, zoom, rotate, light movement and some display modes are all controlled with the mouse. This minimalist, non-obvious philosophy stems from the fact that this viewer was written to help the investigators and was not intended to be used by a general public. Even so, the interface is not hard to learn 
and once learned, it is succinct and quick.

In an attempt to provide for modularity and expandability and to make it as easy as possible for non-developers to add functionality, the main routine that creates the menu bar is very small. It creates the application window and the menu bar and then calls separate routines to add each button to the menu bar and register the list of call backs that will implement that group of functions. A new button (and group of functions) can be added by adding a single line of code to call all the other code needed to implement the function.

The new code can be completely independent of all the other viewer code. The greater the degree to which the new code wants to take advantage of the data structures and utility routines in existing code, the more the coder will have to learn and understand. Also, one who wants to write new code will almost certainly have to know Motif/X11 to do anything useful. But, the new code can be integrated into the viewer structure with a single call in the main routine.

The viewer also provides access to the simplification functions that were the subject of this LDRD. One of the menu bar buttons invokes a pull down menu with assorted simplification functions on it. Not all functions are listed there. Generally speaking, there were two ways in which the simplification functions could interact with the viewer. They could be invoked from the viewer as just described or they could be developed entirely outside the viewer.

If the function is invoked within the viewer, it can either modify the body directly or copy the body and modify the copied body. It can be somewhat easier.to copy the body since the simplification can be followed by fairly high level requests to the viewer to put the new body in a window. If the body is modified directly, then the function must do some careful modifications to the viewing data structures as well. Generally, if the changes are going to be radical or involve many elements of the body, then copying the body is best. If the changes only affect small portions of the body, then it is best to make those changes directly.

If the body has been copied, the function requests the viewer to create a new window and enter the modified body into it. If the function directly modifies the body and the data structures, the function can then just request a redrawing of the window.

Alternately, the simplification function could be developed independent of the viewer. It could read in an ACIS body, manipulate it and write it back out again. The viewer could then be invoked to see what changes had been wrought.

Some simplification functions must be invoked within the viewer since they require the identification of a specific feature on the body. If, for example, you would like to remove a specific hole, rather than all holes of a certain type, it is necessary to invoke the viewer and select the hole visually. There really is no other method of 
identifying.specific features.

\section{Mouse Viewing Functions.}

As mentioned above, the viewing environment is almost exclusively controlled by the mouse. To affect the view in a particular window, move the mouse into the window and then execute the series of clicks and movements desired. Nowhere is the value of a continuous viewing parameter manipulated through sliders or dials. It is always changed via mouse movements in the appropriate window. The discussions that follow assume the mouse is a three button mouse. It is also important to understand that the viewer works with the assumption that you are looking at a particular point, not along a particular direction. This point you are looking at will be called the center-of-interest. It is clearly visualized as the point about which rotations happen.

Pan and Zoom. To pan an object (film critics not withstanding) is to move it in the plane of the screen (i.e. left and right or up and down or some combination). This is done by holding the left and right mouse buttons down at the same time and moving the cursor in the direction that you want the object to move. This action actually moves the center of interest appropriately (in the opposite direction, if you must know) in a plane parallel to the screen and then, as always, keeps the center of interest in the center of the window.

To zoom an object is to move it closer or farther. This is accomplished by moving the mouse with the middle button held down. Movement toward the top of the screen effectively pushes the object farther away. Movement toward the bottom of the screen brings the object closer. Movement from side to side has no affect. This kind of zoom does not change the center of interest. This is a scaled zoom in that as you move closer to the center of interest, your distance from it is multiplied by a factor less than one. Thus, you can never get to the center of interest, just closer and closer. As you move away from it, your distance from it is multiplied by a factor greater than one. So you move away in increasingly larger steps. This feels natural and, indeed, if you wanted to move very far away from a point, it could take a great deal of time if you couldn't increase your stride.

There is also a non-scaled zoom which moves the center of interest directly toward or away from you along the direction you are looking. In this zoom, you stay the same distance from the center of interest and actually move through the scene at a constant rate. The rate is some fraction of your current distance from the center of interest. This is accomplished by holding the control key down as well as the middle mouse button while you move the mouse.

A Word about Clipping. The nature of OpenGl (and most other graphics packages) is such that clipping planes must be specified that remove anything from the picture that is either too far away or too close. It is possible to specify these 
planes to be very, very far away and very, very close to the eye so that everything is always visible. The drawback is that the picture quality degrades (for technical reasons that aren't worth going into here). Consequently, the viewer maintains the far clipping plane to be 5 times as far away as the center of interest and the near clipping plane to be $1 / 20$ th the distance to the center of interest. For this reason, as you zoom closer to the center of interest, you may see parts of the scene disappear from the back and front as they fall behind the new clipping planes. As you zoom out from the center of interest, parts of the scene may suddenly pop back into view.

Rotation. The scene is rotated by holding the left mouse button down and moving the mouse. Movement left and right rotates the scene or object about a vertical axis. Movement up and down rotates the object about a horizontal axis. The axes that it rotates about are the screen axes not the object or world axes. Thus the relationship between the rotational axes and the object axes changes every time a rotation is started. In other words, at the moment the left mouse button is pressed, axes that pass through the center of interest that are parallel to the vertical and horizontal screen axes are used to rotate the scene. These axes remain fixed while the left mouse button is pressed. When the button is released, the axes are forgotten. The next left mouse click establishes new axes about which to rotate.

It may seem that it would be more natural to base the rotation on the object's axes or world axes. However, in practice, it is very hard to look at a scene or object and know which way its axes are currently oriented and, consequently, how the object will move when a rotation is begun. The way rotation is implemented in this viewer, the user can always know how the object will begin to move as a rotation is initiated. This reasoning may not be convincing but trials clearly showed this method as being much less confusing. Even people used to the other way, needed almost no time to adapt and quickly preferred the screen axes orientation.

A second rotational mode is available. The default rotation capability assumes, in effect, that you and the world are stationary and the object is being rotated. Think of it as though the light source is over your shoulder and you are rotating something in your hand. This philosophy makes sense if viewer is used to examine independent bodies. If you wish to move through a larger scene, with many components, you may wish to think of yourself rotating your head in the scene. This kind of rotation is induced by holding the shift key down at the same time as you use the left mouse button.

Recovering From Chaos. Viewing in 3-d is complicated and the opportunities for getting lost are numerous. If you are having trouble getting oriented or just want to start from a known reference frame, you return to the default view that the object was loaded into the viewer with by simultaneously holding the shift and control keys down and then pressing the left button on the mouse. If this view of the object leaves you lost, then something more basic is wrong and it will require a conversation of a more extended give and take than can be accommodated here. 
Moving the Light. In the default setting, the light is positioned over your right shoulder. Using the default rotation, the light will stay there and the object or scene will move. Using other rotations and moves, it is possible to get the light on the far side of an object. You may wish to move the light in this case or you may simply wish to light an object differently. You can move the light by holding the right mouse button down and moving the mouse. Moving the cursor towards the top of the screen swings the light source toward a point directly above you. Moving the cursor towards the bottom of the screen swings the light to a point directly below you. At any place other than the two poles (above and below you) you can swing the light around in azimuth by moving the cursor left and right.It's as though you were moving the sun around the horizon. If you reset the view to the default view as described in the previous section, the light does not reset. You'll have to move it by hand back to some desirable orientation.

Single Face Wire Frame. To draw something "wire frame" is to draw lines along the edges of the polygons that make it up and let it be transparent elsewhere. This function is useful enough that it has been given its own mouse button. If you hold the shift key down and click the middle mouse button, the face that the cursor is on toggles display mode between wire frame and solid. This is very useful for identifying exactly what part of a body a particular face covers. It is also important for seeing how it is decomposed into polygons. It is also quite useful, at times, to be able to see through a face.

\section{The Menu Bar Buttons}

The File Button. The menu that pops up when the file button is pressed is shown at the here. The Load button brings up a file selection dialog box. You can then select an ACIS sat file of your choice. The viewer assumes that every entity in the file is an ACIS BODY. All the bodies are combined into one body and displayed in a new window. The Save button will bring up a file selection box. Once you have selected the file name in which to save it, you will be asked to identify the body you want to save by clicking the mouse on it. The Save Wires button collects all the faces of a body that are currently being display in wire frame mode into a separate body and saves that body into a file that has been selected as in the Load and Save functions. This can be very useful

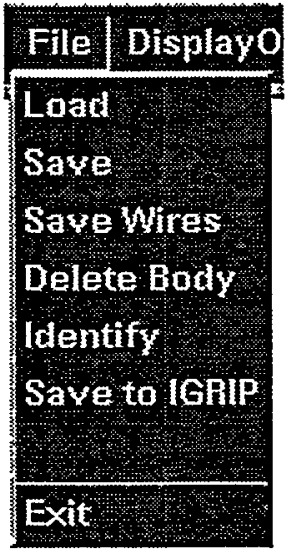
to isolate a group of faces or section of a body that is causing trouble and needs some deeper analysis. It can be that dealing with the rest of the body is extremely time consuming or just obfuscating. This method is a handy way of visually selecting just a portion of the body to work with.

The Delete Body button asks you to identify a body with the mouse (it asks you to confirm it) and then deletes the body and the window from the viewer. The Identify button asks you to click on a body and then displays a window with counts 
for the number of lumps, shells and faces the body has and how many of each of the five types of faces the body has. Also shown is the number of polygons in the body. The Save to IGRIP button writes out the polygonal representation of the body into a file in an IGRIP part file format. This is a sample of how the viewer can be made to translate data from one format to another. The Exit button would appear to be obvious.

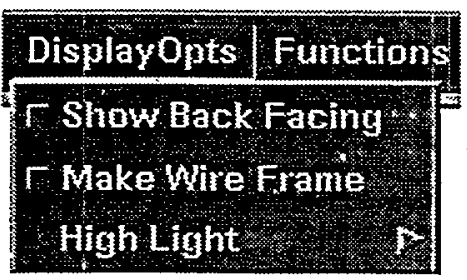

The DisplayOpts Button. This button controls a few options for display modes. When a polygon faces away from you, it is said to be back facing (it's back side is facing you). For any object from the real world, a polygon that represents a portion of its surface can only be seen when it is not back facing. (The proof of this is left as an exercise for the reader.) Consequently, it is a common

practice to not bother drawing backfacing polygons. This makes drawing up to twice as fast while not changing the scene any. However, when you are taking an object apart face by face and peering into it, it can sometimes be very useful to draw the backfacing polygons so you can see the other side of the solid. This viewer defaults to backfacing off but the Show Back Facing button can toggle this display mode.

The Make Wire Frame button will toggle the display mode between wire frame and solid for every face on a body. If a face is already wire frame it is made solid. Thus, if you wanted to eliminate just a few faces from a body (perhaps there is some pathological problem with them) you could select them with the single face wire frame function (a mouse function, see above), use the Make Wire Frame function to toggle the mode for every face and then use the Save Wires function to save all but the troublesome faces.

The High Light button invokes another menu that lists four of the five ACIS surface types (spline surfaces are inexplicably omitted) and a Single Face button. Selecting one of the surface types (cone, plane, torus, or sphere) causes all of the faces with that surface type to be displayed in a unique color. Selecting the surface type again returns those faces to the default yellow. The other button, Single Face, is used to highlight a specific face that you select with the mouse. This has only small benefits visually. The big payoff is that pointers to this face (both the face id in the viewer data structures and the FACE * pointer in ACIS) are noted in global variables in the viewer. Thus if a function requires the identification of a single face, this is a mechanism to do it. In particular, this can be extremely useful when debugging some simplification code.

The Functions Button. This button invokes various simplification functions. The description of the functions is in the main body of this report. Some comments are useful here, however. The Patch Planes button invokes the function to remove arbitrarily shaped holes in planes which is described in third chapter of this report. When this button is pressed, a copy of the body that you indicate is created and passed to the function. The result is presented in a new window. The Patch A Plane 
button does the same for a single planar face that you indicate. Also, for this function, the simplification is done directly on the body you select. No copy is made.

The Fill Holes button invokes the function to remove circular holes from the entire body that fit the heuristics described in the second chapter. The body you select is copied and processed and the result is viewed in a new window. The Fill Specific Hole button asks you to select a circular hole by clicking on the wall of the cylinder. The specific hole is removed directly (no copy going on) and the result redisplayed in the original window.

The Remove Chamfers and Remove Fillets buttons do not work yet as the functionality they represent has not been integrated into the viewer. You must invoke these as external functions at this time.

The functionality of the Remove Face button is not described elsewhere since it is such a low level, brute force simplification technique. After clicking on this button, you select a face of any sort by clicking on it with the mouse and it is directly removed from the body.

The Remove Internals and Raise Shelves buttons invoke the functionality described in the fifth and fourthth chapters respectively. In each case, the body is copied and processed and the results show in a new window.

The Other Stuff Button. This button is included as an example of how to add buttons and functionality to the viewer. The pull down menu invoked by this button does very little. Try it sometime. The point is that working with Motif is difficult and the code for this button serves as a simple, straight forward example on which to build.

The Set Parms Button. This button brings up a window in which the parameters for the various simplification functions can be set. For example, the maximum radius of holes that will be removed or the difference between shelves. The values are specified in a text editable field. Simply change the values. It is not necessary to hit return. 
This page intentionally left almost blank. 


\section{DISTRIBUTION:}

1 MS $0188 \quad$ LDRD office, 4001

$1 \quad 1002 \quad$ P. J. Eicker, 15200

$1 \quad 1004$ R. Harrigan, 15221

101004 Peter Watterberg, 15221

$1 \quad 9018$ Central Technical Files, 8940-2

$2 \quad 0899 \quad$ Technical Library, 4916

10612 Review \& Approval Desk, 4912 For DOE/OSTI

10161 . Patent and Licensing Office, 11500 